\title{
ARTICLE
}

Cite this: DOI: 10.1039/C4RA12431F

Accepted 1st December 2014

DOI: $10.1039 / C 4 R A 12431 F$

www.rsc.org

\section{High-Throughput Microfluidic Platform for Adherent Single Cells Non-Viral Gene Delivery}

\author{
P. Occhetta, ${ }^{a}$ C. Malloggi, ${ }^{b}$ A. Gazaneo, ${ }^{a}$ A. Redaelli, ${ }^{a}$ G. Candiani, ${ }^{b c d}$ M. Rasponi ${ }^{a *}$
}

The widespreading of gene therapy as therapeutic tool relies on the development of DNAcarrying vehicles devoid of safety concerns. Opposite to viral vectors, non-viral gene carriers are promising in this perspective, although their low transfection efficiency leads to the necessity of further optimizations. Aiming at overcoming the limitation of traditional macroscale approaches, mainly consisting in time-consuming and simplified models, a microfluidic strategy was developed for transfection studies on single cells in a highthroughput and deterministic fashion. A single cell trapping mechanism was implemented based on dynamic variation of fluidic resistances. At this purpose, we conceived a roundshaped culture chamber integrated with a bottom trapping junction which modulates the hydraulic resistance. Several layouts of the chamber were designed and computationally validated for the optimization of the single cell trapping efficacy. The optimized chamber layout was integrated in a polydimethylsiloxane (PDMS) microfluidic platform presenting two main functionalities: (i) 288 chambers for trapping single cells, (ii) a serial dilution generator provided with chaotic mixing properties, able to deliver to chambers both soluble factors and non-diffusive particles (i.e. polymer/DNA complexes, polyplexes) under spatio-temporally controlled chemical patterns. Devices were experimentally validated and allowed for trapping individual human glioblastoma-astrocytoma epithelial-like cells (U87-MG) with a trapping efficacy of about $40 \%$. Cells were cultured within the device and underwent preliminary transfection experiments using $25 \mathrm{kDa}$ linear polyethylenimine (IPEI)-based polyplexes, confirming the potentiality of the proposed platform for the future high-throughput screening of gene delivery vectors and the optimization of transfection protocols.

\section{Introduction}

Gene therapy has opened new scenarios in treating diseases compared to conventional therapeutic strategies by modifying and controlling protein expression of target cells through the introduction of specific genetic material ${ }^{1}$. Being DNA and RNA negatively charged biopolymers, the direct delivery of naked nucleic acids to cells is the least efficient approach. To overcome such a limitation, recent advances in materials science and biotechnology have enabled the development of a variety of systems for the delivery of nucleic acids to cells. Non-viral vectors display striking advantages over viral vectors (i.e. lower cytotoxicity, lower to no immunogenicity and greater ease of preparation and handling) ${ }^{2}$; on the other hand, they still require further optimization in terms of transfection efficiency ${ }^{3,4}$. Non-viral gene delivery vectors are inherently cationic at physiological $\mathrm{pH}$, thus they spontaneously organize with nucleic acids in nano- and micro-particles ${ }^{5}$. In this scenario, the research for optimized and innovative non-viral gene vectors through conventional bench-top systems -such as cell cultures in multiwell plates- is time-consuming, demands relatively large quantities of materials and allows for simultaneously testing only a limited number of materials and parameters. Moreover, such traditional approaches rely on averaging data from heterogeneous cell populations, thus being suboptimal for assessing individual cell responses to exogenous stimuli, such as transfection ${ }^{6}$. In the last years, great interest has thus been focused on single cell analysis and screening, which represents a promising tool aiming at pursuing the direct and deterministic control over cause-effect relationships guiding cell behavior ${ }^{7}$. However, the analysis on single cells still presents a variety of challenges. First, in order to obtain statistically significant responses, the ability to handle a large number of individual cells in a high-throughput fashion is crucial ${ }^{8}$. Moreover, the capability of reliably allocate individual cells in well-defined locations is crucial for subsequent analyses, often implying the tracking of cells over the culture period ${ }^{9}$. Finally, the typical size of features needed to address individual cells demands more strict requirements 
with respect to standard culture tools in terms of sensitivity, selectivity and temporal resolution ${ }^{10}$.

Recent advances in microtechnologies and microfabrication are offering increasing cues for addressing these challenges. Moreover, the reduction of scale leads to unique fluidic phenomena, e.g. the increasing of surface-to-volume ratios, which results in shorter reaction times, higher efficiencies, and lower material consumption ${ }^{11}$. In the last few years, several strategies have been proposed for single cell manipulations within microfluidic devices, being exploited in a number of applications in the field of cell biology ${ }^{12}$. However, these approaches for trapping single cells require complex and expensive fabrication procedures due to the need of multilayered devices ${ }^{13,14}$. As an alternative, small trapping units were recently proposed ${ }^{15-19}$ to block cells along their fluidic path; however their integration with chambers for subsequent culture is not trivial. An interesting approach allowing for trapping and culturing single cells within a microfluidic devices based on a variable resistance mechanism -the cells functioning as fluid stopper once trapped-at the outlet of a culture chamber was recently reported by Hong and colleagues ${ }^{9}$. However, the requirement in terms of feature sizes did not allow for using conventional photolithographic techniques thus preventing design reproducibility (chemical etching of SU8 photoresist was used to obtain feature sizes of about $3 \mu \mathrm{m}$ ).

Herein, based on a similar principle, a low cost and single-layer polydimethylsiloxane (PDMS) microfluidic platform is presented for isolation and culture of a large number of individual adherent cells into defined spatial configurations. The single cell trapping unit was optimized aiming at overcoming issues on fabrication reproducibility, while maintaining high trapping efficacy. Moreover, the upstream integration of a serial dilution generator (SDG) based on a chaotic mixing scheme allowed for the first time to combine the capability of trapping single cells with the ability of delivering them both soluble factors and non-diffusive particles (i.e. polymer/DNA complexes, polyplexes) under spatio-temporally controlled chemical patterns. Herein, we provide the first proofof-principle demonstration with a typical transfectant that our microfluidic device can be used proficiently for the forthcoming optimizations of the whole variety of gene delivery vectors. Such platform may thus represent a powerful tool for the implementation of non-viral gene transfection protocols allowing for (i) testing multiple conditions in an automatic and high-throughput fashion, while (ii) directly monitoring gene transfer efficiency and toxicity through "on-off" direct investigations over single cell behavior.

\section{Materials and methods}

\section{Single cell trapping mechanism}

A microfluidic unit layout was designed aiming at trapping single adherent cells while ensuring enough space for their subsequent growth (Fig1a-c). A single cell trapping mechanism was thus implemented exploiting an automatic variation of hydraulic resistance within the chamber ${ }^{9}$. In details, a circular culture chamber $(\Phi=250 \mu \mathrm{m}, \mathrm{h}=25 \mu \mathrm{m})$ was conceived and provided with two connections to a main fluidic path: (i) an upstream wide opening, which allows for both transportation of cells and diffusion of media/conditioning factors, and (ii) a downstream trapping junction which modulates the hydraulic resistance. The mechanism of trapping is schematically depicted in Fig.1c. Briefly, a cell entering the chamber follows the flow stream, eventually reaching the trapping junction (blue trajectory); the subsequent partial, or total, occlusion of the trap causes an increase in terms of hydraulic resistance, and, in turn, a reduction of inflow within the chamber (blue arrow), thus preventing the entrance of further cells (red trajectory). (a) Trapping unit layout

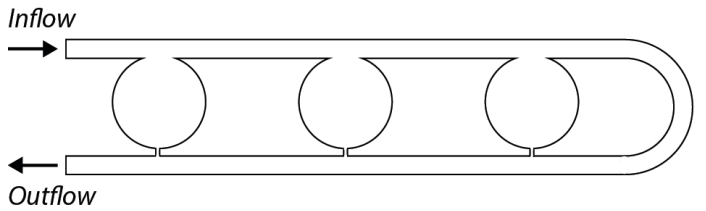

(b) Chamber dimensions

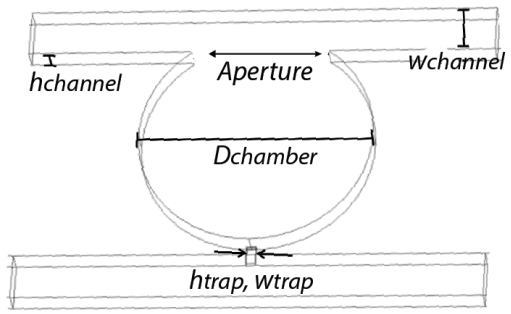

(c) Single cell trapping mechanism

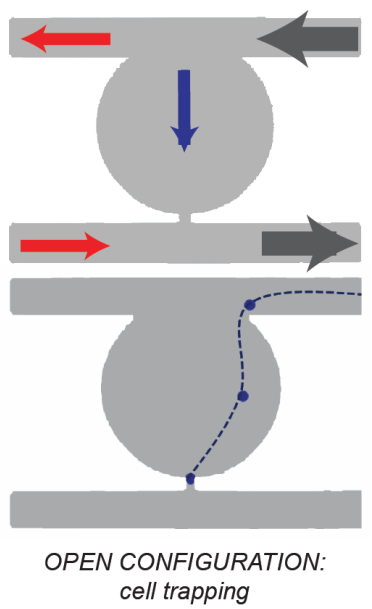

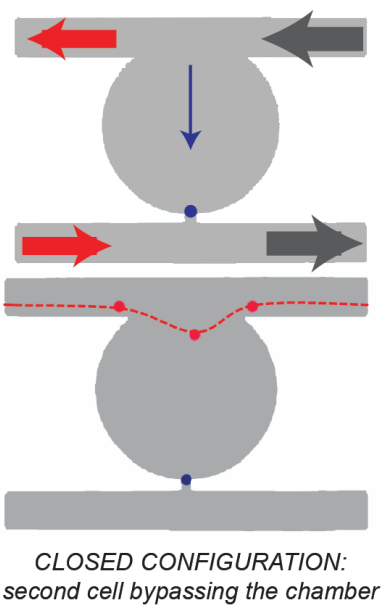

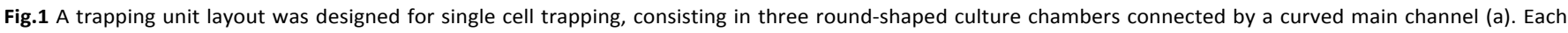

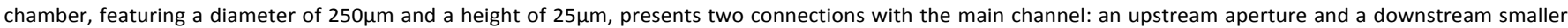

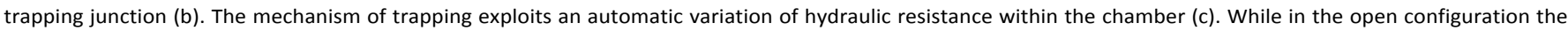

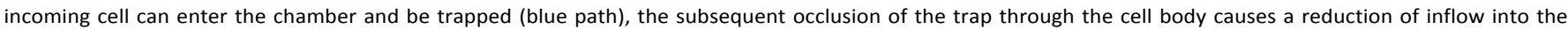
chamber (blue arrows), thus inhibiting the entrance of further cells (red trajectory). 


\section{Computational optimization of the chamber geometry for single} cell trapping

The geometry of the chamber was optimized for single cell trapping by means of Computational Fluid Dynamic modeling (CFD, Comsol Multiphysics, see Supplementary Information). For ease of microfabrication, the heights of fluidic (consisting on flow channels and culture chambers) and trapping layers were fixed at $25 \mu \mathrm{m}$ and $5 \mu \mathrm{m}$, respectively. Finite element analyses were performed on a $3 \mathrm{D}$ model of a fluidic unit, consisting of three culture chambers (provided with trapping elements), and a curved main channel (upstream and downstream) (Fig.1a). Different unit layouts were analyzed, all potentially compatible with standard fabrication techniques, aiming at achieving the highest trapping efficacy, while maintaining the selectivity for individual cell trapping. In details, eight geometries were considered, as combinations of four different widths for the fluidic channels $(25,50,75$ and $100 \mu \mathrm{m})$ and two widths for the trapping junctions (10 and $15 \mu \mathrm{m}$ ) (see Supplementary information Table SI1). Furthermore, each configuration was characterized by either the absence or presence of trapped cells -namely open and occluded configurations, respectively-, modeled as solid spheres, featuring diameters comparable with trap widths (10 and $15 \mu \mathrm{m}$ ), slightly intersecting the boundaries in proximity of the trapping element. The comparison among numerically calculated flow rates entering the chamber in both open and occluded configurations resulted in an optimal configuration in terms of trapping efficacy; featuring $10 \mu \mathrm{m}$ wide trapping junctions and $50 \mu \mathrm{m}$ wide main channel (see Supplementary Information, Fig.SI2). The optimal geometry was thus integrated in the final platform and used for experimental validations.

\section{Design of the chaotic mixer serial dilution generator}

A serial dilution generator (SDG) provided with chaotic enhanced mixing features was integrated to the microfluidic platform for mixing both soluble factors (i.e. growth factors) and non-diffusive assemblies (i.e. polyplexes), splitting an initial concentrated solution into 6 linear dilutions (from $0 \%$ to $100 \%$ in steps of $20 \%$ ). The chaotic mixer SDG was designed based on a resistive flow model consisting of symmetric microfluidic networks ${ }^{20}$ composed of thin channels $(60 \mu \mathrm{m}$ wide and $25 \mu \mathrm{m}$ tall) whose lengths were dimensioned to allow diffusive mixing of soluble factors. Furthermore, aiming at mixing polyplexes which feature diameters ranging from $100 \mathrm{~nm}$ to $1 \mu \mathrm{m}^{21-24}$, staggered herringbone (HB) grooves were integrated on top of the channels ${ }^{25}$. Each HB was designed $24 \mu \mathrm{m}$ wide and $9 \mu \mathrm{m}$ high, being an $\mathrm{HB}$ unit constituted of eight HBs changing their orientation between half cycles. For Reynolds numbers $(\mathrm{Re})$ ranging between 0.1 and $10(\mathrm{Q}=0.255$ $25.5 \mu \mathrm{L} / \mathrm{min}$ ), the mixing efficiency was validated for nondiffusive particles through finite element analysis by means of Computational Fluid Dynamic models (Comsol Multiphysics), as detailed in the Supplementary Information. The addition of HB grooves demonstrated to enhance the mixing of nondiffusive entities and, in the same time, the need of a smaller footprint in the device area (see Fig.SI3). (a) Device layout

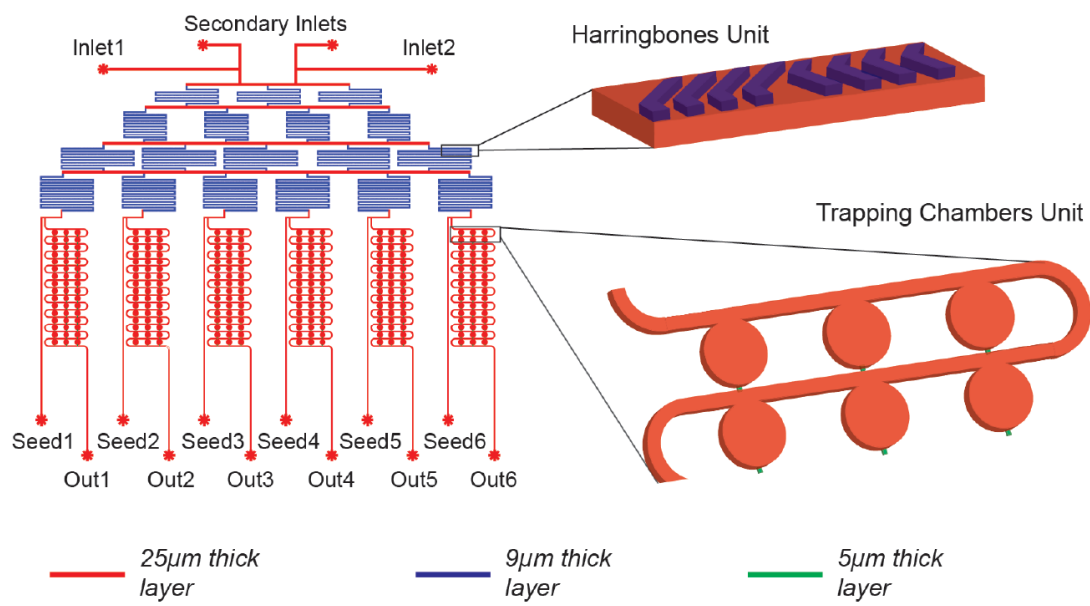

(b) PDMS microfluidic platform

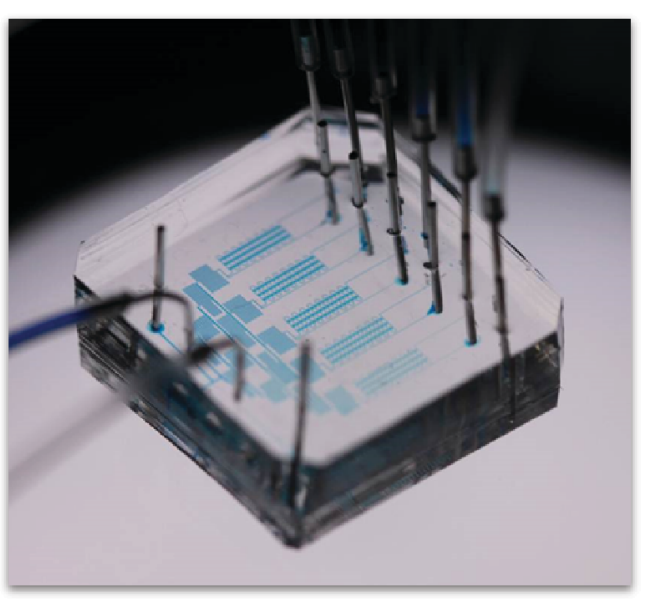

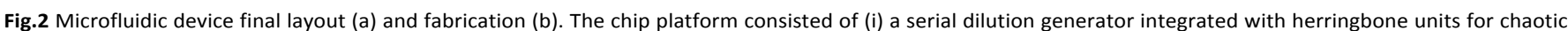

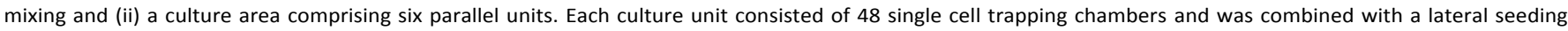

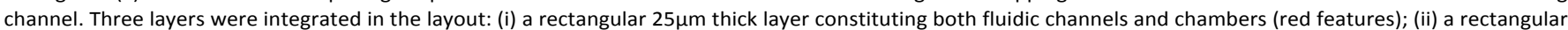

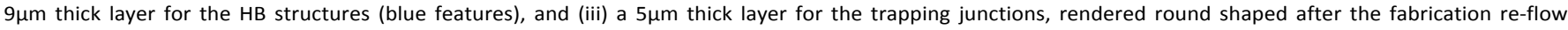
process (green features). 


\section{Fabrication of the microfluidic platform}

The final chip layout was designed integrating the computationally optimized versions of both the trapping chamber and the HBs mixing unit. In details, the final microfluidic platform featured an upstream chaotic mixer serial dilution generator, which independently delivered six linear dilutions to six downstream culture units (Fig.2a).

Each culture unit consisted in the repetition of 48 single trapping chambers (trap size $10 \mu \mathrm{m}$ ), connected through a main channel $50 \mu \mathrm{m}$ wide, and integrated with a lateral seeding channel. Two secondary inlets were finally included in the layout to facilitate the medium change operations. The layout was finalized with CAD software (AutoCAD, Autodesk Inc.) and the corresponding master mold was realized through standard photolithography techniques ${ }^{26}$. In particular, three layers were integrated: (i) a $25 \mu \mathrm{m}$ thick layer constituting both fluidic channels and chambers (Fig.2a, red features); (ii) a $9 \mu \mathrm{m}$ thick layer for the HB structures, positioned on the top of the previous one (Fig.2a, blue features), and (iii) a $5 \mu \mathrm{m}$ thick layer for the trapping junctions, reflowed to achieve a rounded crosssection (Fig.2a, green features).

Microfluidic devices (Fig.2b) were obtained by replica molding of PDMS (Sylgard ${ }^{\mathbb{B}}$ Dow Corning) on the master mold. Briefly, PDMS was cast on the mold in ratio $10: 1 \mathrm{w} / \mathrm{w}$ (pre-polymer to curing agent), degassed and cured at $80^{\circ} \mathrm{C}$ for $3 \mathrm{hr}$. Input and output ports were obtained through a $0.5 \mathrm{~mm}$ biopsy puncher (Harris Uni-Core ${ }^{\mathrm{TM}}$ ) and the PDMS chip was finally permanently bonded to a glass slide $(25 \times 75 \times 1 \mathrm{~mm})$ upon a 1 min of air plasma treatment (Harrick Plasma).

\section{Experimental validation of the chaotic mixer serial dilution generator}

The dilution patterns generated by the chaotic mixer serial dilution generator were experimentally assessed for both soluble and non-diffusive species and compared with those obtained through an equivalent diffusive SDG featuring nongrooved channels.

In details, for testing the diffusive mixing of soluble species, a blue dye solution (food color) was pumped into one inlet at a flow rate of $36 \mu \mathrm{L} / \mathrm{hr}$ while distilled water $\left(\mathrm{dH}_{2} \mathrm{O}\right)$ into the other one. Upon the achievement of a steady state condition, three samples of $20 \mu \mathrm{L}$ of dilution were collected from each outlet and the $\mathrm{OD}_{570 \mathrm{~nm} \text { for }}$ each dilution was quantified by means of a spectrophotometer (NanoDrop 2000c, Thermo Scientific, Wilmington, USA). For both SDG configurations, experiments were carried out on three different devices $(n=3)$ and compared with expected values.

The evaluation of mixing of non-diffusive assemblies was performed for both chaotic and diffusive SDGs by pumping DNA/polymer complexes through the cmSDG. Briefly, polyplexes were prepared at room temperature (r.t.) by adding ZsGreen plasmid (Clontech Laboratories, Inc., CA, USA) to $25 \mathrm{kDa}$ linear polyethylenimine (IPEI, PolyScience) (final plasmid concentration in the transfection suspension: $40 \mathrm{ng} / \mathrm{mL}$ ) in $150 \mathrm{mM} \mathrm{NaCl}$ at $\mathrm{N} / \mathrm{P} 40 . \mathrm{N} / \mathrm{P}$ is defined as the molar ratio of the amino groups of the cationic polymer used to complex the phosphate groups of a given amount of DNA. Polyplexes finally featuring an average hydrodynamic diameter of $1.37 \pm 0.27 \mu \mathrm{m}$, as determined by Dynamic Light Scattering (DLS, Zetasizer Nano ZS instrument) were left to form for $20 \mathrm{mins}$ at r.t. before use. The final polyplex suspension was pumped in the right inlet at a flow rate of $3.75 \mu \mathrm{L} / \mathrm{hr}$, corresponding at $\mathrm{Re}=1$, while $150 \mathrm{mM} \mathrm{NaCl}$ into the left one. After the achievement of a steady state condition, three samples of $1.5 \mu \mathrm{L}$ of dilution were collected from each outlet and DNA concentration was assessed by measuring the $\mathrm{OD}_{260}$ by means of a spectrophotometer (NanoDrop 2000c). For both the SDG configurations, the results were collected as the average of four devices $(n=4)$ and compared with expected values.

\section{Cell expansion}

U87-MG (human glioblastoma-astrocytoma epithelial-like) cell line was purchased from the American Type Culture Collection (ATCC). Cells were cultured at $37^{\circ} \mathrm{C}$ in a humidified atmosphere containing $5 \% \mathrm{CO}_{2}$ in Dulbecco's Modified Eagle Medium (DMEM, Sigma-Aldrich) supplemented with $1 \mathrm{mM}$ sodium pyruvate, $10 \mathrm{mM}$ HEPES buffer, $100 \mathrm{U} / \mathrm{mL}$ penicillin, $0.1 \mathrm{mg} / \mathrm{mL}$ streptomycin, $2 \mathrm{mM}$ glutamine, and $10 \%(\mathrm{v} / \mathrm{v})$ fetal bovine serum (FBS) (hereafter referred as to complete DMEM).

\section{Single cell trapping and culturing within microfluidic device}

Preliminary biological experiments were carried out on the computationally optimized platform to evaluate the trapping efficiency and the device compatibility for adherent single cell cultures.

Before cell seeding, microfluidic devices were sterilized by autoclaving $\left(121^{\circ} \mathrm{C}, 20 \mathrm{mins}\right.$, wet cycle) and subsequently dried overnight at $80^{\circ} \mathrm{C}$. To favor cell adhesion on the glass substrate, devices were air plasma treated for $5 \mathrm{mins}$ to increase the hydrophilicity and subsequently perfused overnight at $37^{\circ} \mathrm{C}$ with a $0.01 \%(\mathrm{w} / \mathrm{v})$ Poly- $L$-Lysine (PLL) solution (SigmaAldrich) at a flow rate of $30 \mu \mathrm{L} / \mathrm{hr}$. After rinsing in PBS, complete DMEM was perfused from the two main inlets.

Trypsinized U87-MG cells were diluted to a concentration of $2 \times 105$ cells $/ \mathrm{mL}$ and a single cell suspension was obtained by means of a cell strainer $(40 \mu \mathrm{m}$, Falcon $\mathbb{R})$. Cells were then simultaneously seeded within the six culture units by perfusing the cell suspension through the seeding channels at a constant flow rate of $0.2 \mu \mathrm{L} / \mathrm{min}$ for $5 \mathrm{mins}$ and subsequently rinsing with fresh medium. Phase contrast images were then acquired by means of Leica DMIRD inverted microscope and the trapping dynamics was further monitored by means of a high-speed camera (Phantom Miro). Single cell trapping efficacy was calculated as the percentage of chambers containing a single cell over the total number of chambers per culture unit. Moreover, to define the specificity of the proposed approach the percentage of chambers containing either i) multiple cells blocked within a trap or ii) one or more cells out of the trap was assessed and compared with the single cell trapping efficacy (See Fig.SI5). Single cell trapping data were collected from experiments as the average of seven devices $(n=7)$. 
Upon cell injection, seeding channels were clamped and a continuous flow rate of $2 \mu \mathrm{L} / \mathrm{hr}$ per culture line was imposed. The microfluidic platform was incubated under standard culture conditions and periodically monitored for assessing cell adhesion and proliferation.

\section{Cell transfection assessment}

The single cell microfluidic platform was exploited for preliminary transfection experiments. In details, single cells were seeded within the microchambers as previously described and allowed adhering overnight. Twenty-four hrs after seeding, the culture medium was replaced with polyplex-containing fresh medium (20ng/ $\mu \mathrm{L}$ of $\mathrm{ZsGreen}$ plasmid). The microfluidic platform was then incubated at $37^{\circ} \mathrm{C}$ up to 3 days. Transfected cells were periodically visually inspected and photographed with Zeiss Axioplan and Leica DMIRD inverted microscope for observing ZsGreen plasmid expression and cell morphology, respectively. Cells were finally fixed by perfusing $4 \%$ paraformaldehyde for 20mins, and after rinsing in PBS cell nuclei were stained with DAPI. Fluorescence images were acquired directly through the optically transparent glass-PDMS devices.

\section{Results}

\section{Validation of the chaotic mixer serial dilution generator}

The chaotic mixer serial dilution generator efficacy in mixing both soluble factors and non-diffusive particles was experimentally assessed and compared with a diffusive SDG.

To evaluate the performance in mixing of soluble factors, a blue dye solution was perfused through the device and allowed to mix with $\mathrm{dH}_{2} \mathrm{O}$, as exemplified in Fig.2b. For both the SDG configurations, the absorbance of the six dilutions collected from the outlets were measured to match the expected linear distribution with coefficients of determination equal to $\mathrm{R}^{2}=0.9891$ and $\mathrm{R}^{2}=0.9948$ for the diffusive $\mathrm{SDG}$ and for the chaotic SDG, respectively (Fig.3a).

To evaluate the mixing of non-diffusive particles, lPEIbased polyplexes were perfused into the device. The results obtained by means of the chaotic mixer confirmed the generation of a linear partition of the injected vectors (Fig. $4 \mathrm{~b}$ ), matching the expected linear distribution $\left(\mathrm{R}^{2}=0.9594\right)$. In contrast, constant concentrations of DNA were measured among the three outlets corresponding to $100 \%, 80 \%$ and $60 \%$ of the diffusive SDG, while no DNA was detected for the remaining outlets, thus demonstrating that no mixing occurred within the device (Fig.3b).

\section{(a) Soluble factor mixing}

\section{Chaotic SDG}
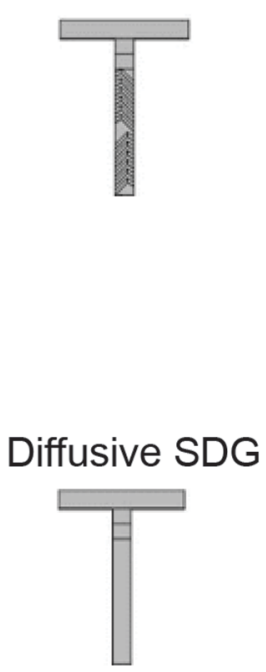
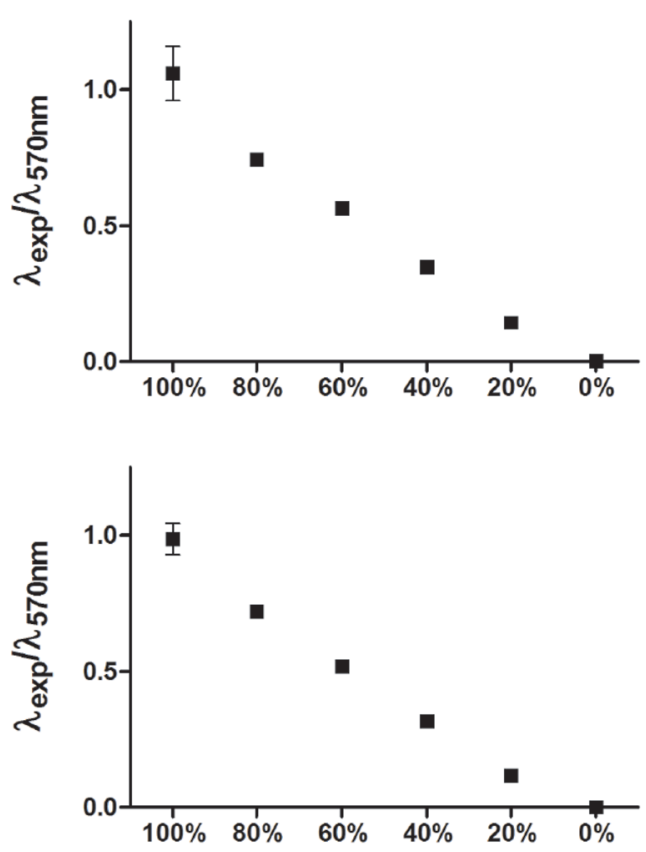

\section{(b) Non-diffusive particles mixing}
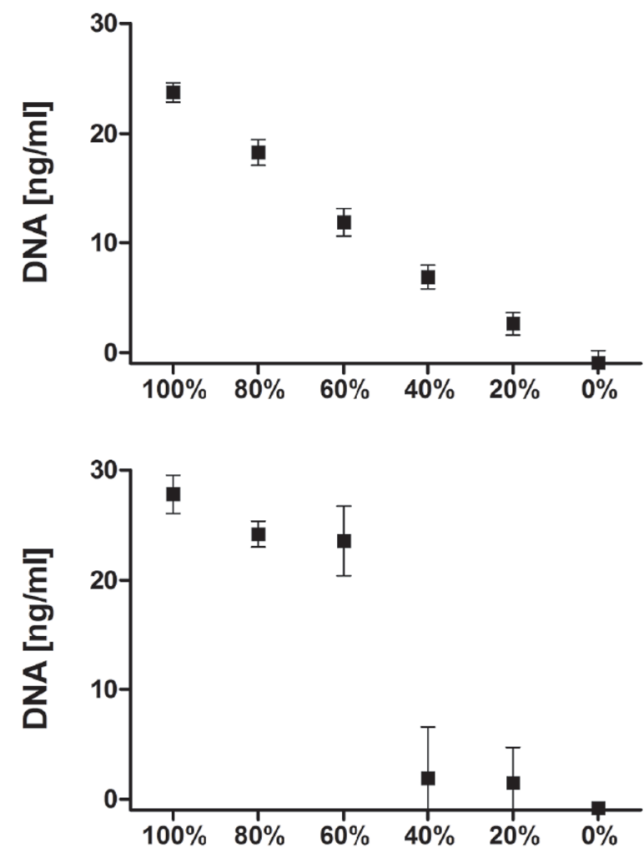

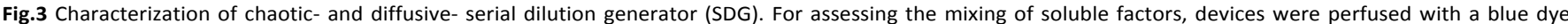

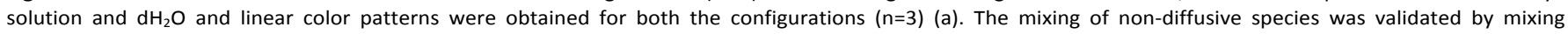

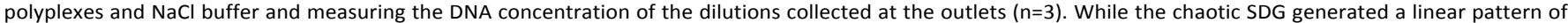
non-viral vectors $\left(R^{2}=0.9594\right)$, within the diffusive SDG no mixing occurred $(b)$. 
(a) Single cell trapping dynamics

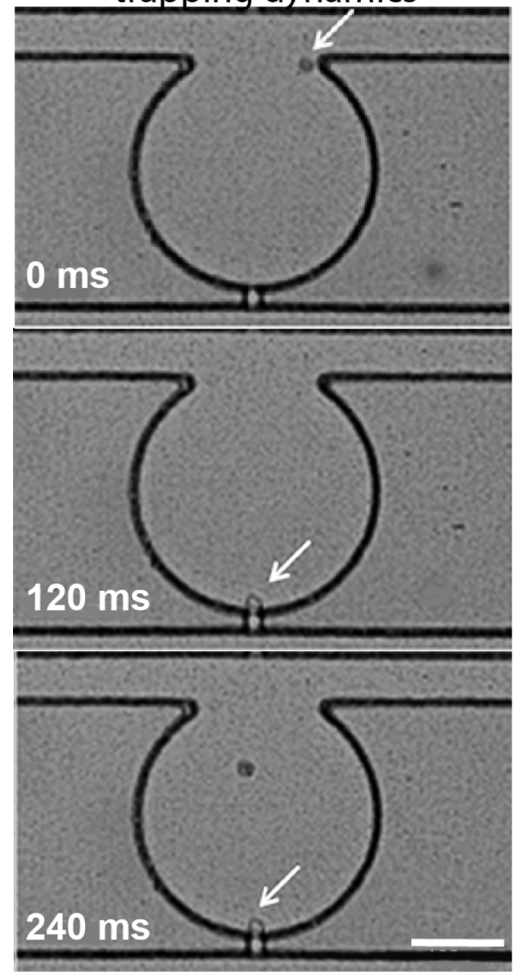

(b) Single cell trapping efficiency

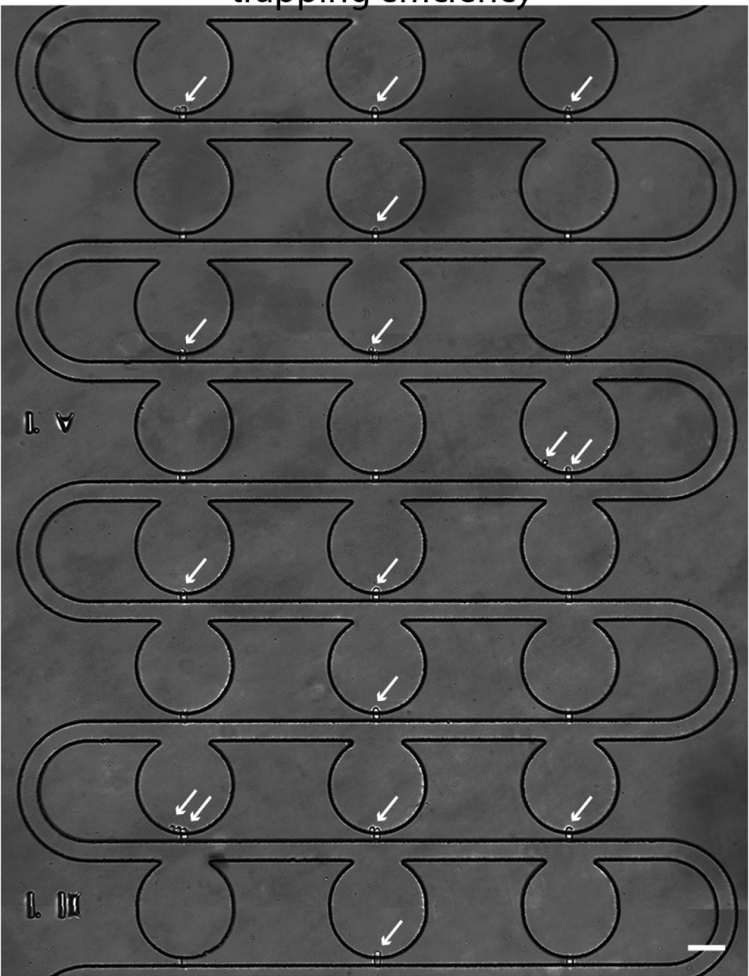

(c) Cell proliferation

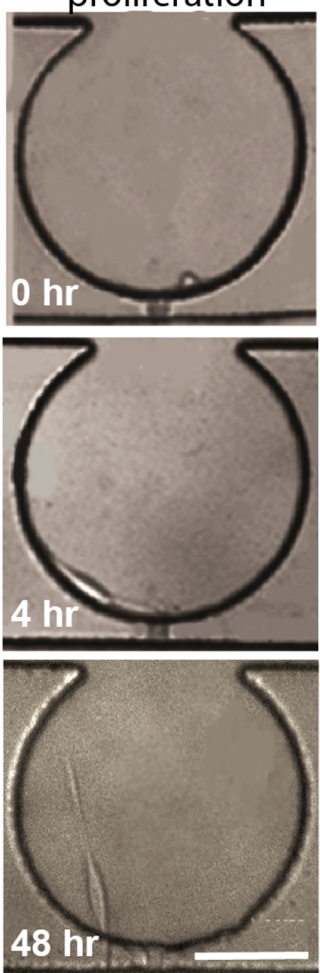

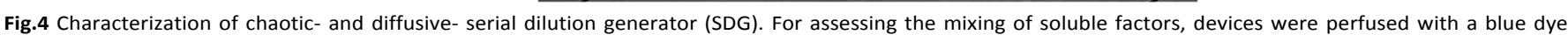

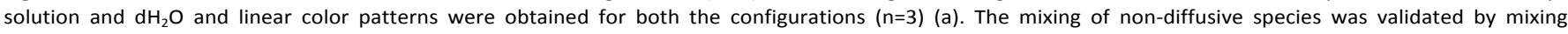

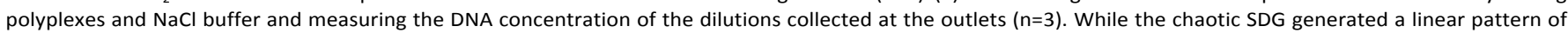
non-viral vectors $\left(R^{2}=0.9594\right)$, within the diffusive SDG no mixing occurred $(b)$. 


\section{Single cell trapping and culturing within microfluidic device}

The biological validation of the optimized platform was carried out by seeding the device with U87-MG cells $(\Phi=15.4 \pm 2.5 \mu \mathrm{m})$, that were kept in culture over 5days. The dynamics of the cell trapping mechanism and the kinematics of cells while being trapped were monitored by means of a high-speed camera (Fig.4a). Noteworthy, the overall percentage of chambers filled with cells per unit was $47.8 \pm 12.2 \%$, whose $39.8 \pm 6.2 \%$ chambers featured single cell entrapped, $6.2 \pm 4.2 \%$ multiple cells entrapped and $1.7 \pm 2.4 \%$ one or more cells out of trap (Fig.5). The single cells seeded within the device took $4 \mathrm{hrs}$ to adhere completely to the PLL-functionalized substrate (Fig.4c), whereas cell proliferation was apparent only $48 \mathrm{hrs}$ after seeding (Fig.4c). Most important, the cells did generally maintain high viability, as highlighted by the proper elongated phenotype shown, for up to 5 days in culture.

\section{Cell transfection assessment}

To assess the potentiality of the microfluidic platform as a tool to perform transfection experiments, $25 \mathrm{kDa} / \mathrm{PEI}$ was chosen as one of the most popular and effective polymeric gene delivery vectors, and thus used as a standard in transfection experiments $^{24}, 27,28$. IPEI-based polyplexes were delivered to the trapped single cells and transgene-driven protein expression was observed by fluorescence microscope inspection. Fig.6 shows a fluorescent image of a transfected ZsGreen-expressing cell detected $12 \mathrm{hrs}$ from the exposure. The elongated morphology indicated the complete adhesion of the cell to the substrate and thus its viability. These results, although preliminary, pave the way for the use of such microfluidic device for the high-throughput screening of gene delivery vectors and the optimization transfection protocols.

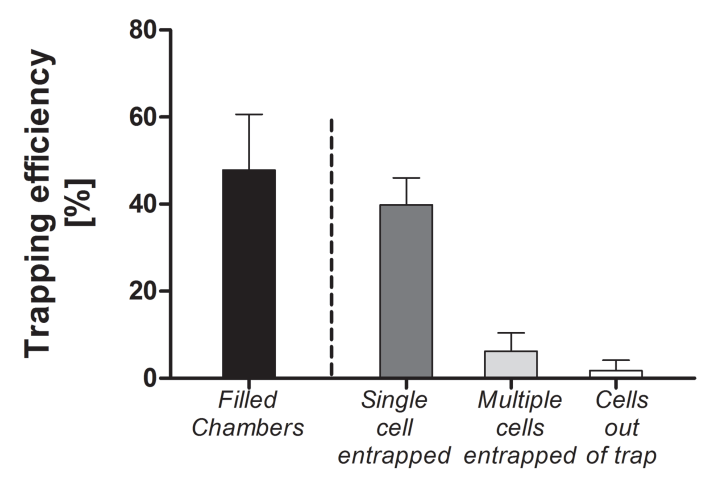

Fig.5 Upon U87-MG cell seeding, the overall percentage of chambers filled with cells per culture unit was $47.8 \pm 12.2 \%$, whose $39.8 \pm 6.2 \%$ chambers featured single cell entrapped, $6.2 \pm 4.2 \%$ multiple cells entrapped and $1.7 \pm 2.4 \%$ on or more cells out of trap $(n=7)$.

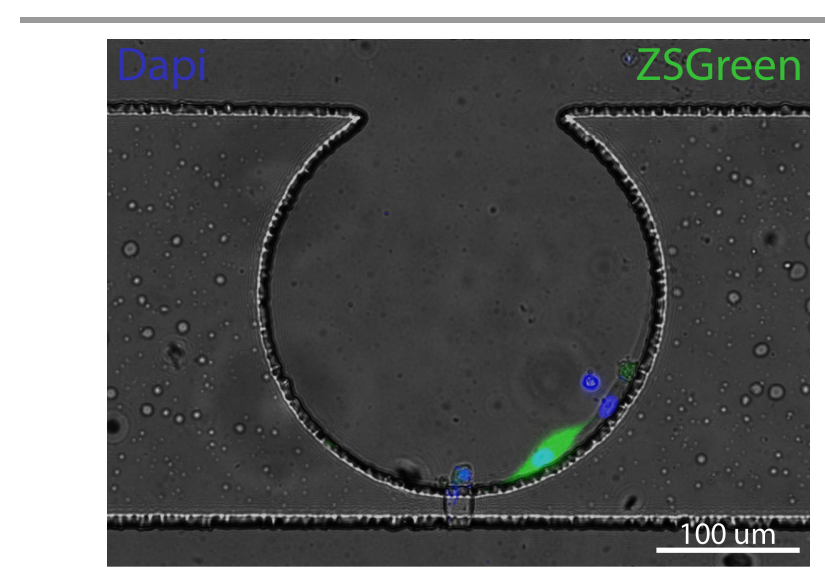

Fig.6 Microscopic observation of a single U87-MG cell transfected with IPEI/ZsGreen plasmid complexes at N/P40. The ZsGreen fluorescence (green) and the DAPI staining (blue) were overlaid onto a contrast image.

\section{Discussion and conclusions}

Aiming at the optimization of non-viral based gene delivery strategies ${ }^{6}$, the possibility to work at a single cell level allows to directly and deterministically control the efficacy of different transfection stimuli on cell behavior. Although conventional techniques have been exploited for isolating single cells (i.e. flow cytometry, capillary electrophoresis) ${ }^{29}$, they still suffer from a poor control over the cellular microenvironment. Conversely, microfluidics has proven to be the leading tool for single cell analysis allowing for precise fluid and cell manipulation while featuring a scale-size comparable to the cellular level ${ }^{10,30}$. In addition, microfluidic assays can be easily parallelized for testing several parameters at a time within the same platform. These advantages make them suitable tools for intensive studies aiming at developing optimized and innovative non-viral gene delivery systems in a fast and effective fashion, thus overcoming the limitations of conventional bench-top systems.

In this regards, we computationally designed and experimentally validated a PDMS microfluidic platform for high-throughput single cell analysis, coupling the ability to handle single cells to defined spatial configurations while precisely tailoring the microenvironment around them. The device integrated two functional units: (i) a cell culture area for single cell compartmentalization, consisting of six independent culture units, and that are individually addressable through the lines of (ii) an integrated chaotic serial dilution generator for further conditioning them through spatio-temporally controlled patterns of non-diffusive particles (i.e. polyplexes).

To date, existing approaches for trapping single cells within microfluidic devices mainly require either complex actuation systems or expensive fabrication procedures difficult to be replicated in reproducible way ${ }^{13,14}$. Such approaches, which allowed for trapping single cells by actuating integrated valve systems $^{31}$ or exploiting physical obstacles as traps ${ }^{14}$, have a drawback in terms of device fabrication. Recently a new approach was presented to successfully isolate single cells within small trapping elements ${ }^{15,19}$. While this method was based on trap configurations geometrically optimized for achieving high trapping efficiency, it resulted not suitable for adherent cell cultures, lacking in sufficient space for cell spreading and subsequent proliferation. Thus, the ability to combine both single cell trapping and culturing capabilities in 
separated chambers still remains an open issue. Among others, an interesting solution has been recently proposed by Hong $\mathrm{S}$ et al. ${ }^{9}$, who integrated an easy to handle single cell trapping mechanism, based on passive variation of hydraulic resistances, directly with compartmentalized culture chambers ${ }^{15}$. Although promising, this approach was shown to require nonconventional fabrication approaches to obtain suitable trap dimensions (down to $3 \mu \mathrm{m}$ ). Starting from the same principle, we here optimized the trapping chamber geometry in the attempt to match both time reproducible fabrication and trapping efficacy requirements. Culture units were considered, consisting of three fluidically connected round-shaped culture chambers $(\Phi=250 \mu \mathrm{m}, \mathrm{h}=25 \mu \mathrm{m})$ integrated with a downstream trapping junction $(\mathrm{h}=5 \mu \mathrm{m})$. Eight geometries were systematically investigated through CFD modeling to maximize the trapping efficacy, while maintaining the selectivity for individual cell trapping over a broad range of cell diameters. The comparison of numerically calculated flow rates entering the chamber in both open and occluded configurations yielded an optimal configuration in terms of trapping efficacy, featuring $10 \mu \mathrm{m}$ wide trapping junctions and $50 \mu \mathrm{m}$ wide main channel. It is worth noting that such optimized configuration featured sizes compatible with standard photolithographic techniques ${ }^{26}$, thus overcoming the main limitation of the previous work ${ }^{9}$.

The experimental validation further confirmed the computational results. In detail, the proposed trapping mechanism allowed achieving high single cell trapping efficiency (up to $40 \%$ ), having all six independent culture units simultaneously filled with individual U87-MG cells within few minutes. A flow rate of $0.2 \mu 1 / \mathrm{min}$, corresponding to Re 0.1 , was chosen to seed the device. Such value was found to be a good compromise between selectivity for capturing single cells and seeding time. Indeed, the use of higher flow rates (around Re 1) increased the possibility for multiple cells to enter the same chamber. At the same time, these flow rates yielded higher pressure drops across traps ends, causing cells deformation and their subsequent escape through the traps, thus in turn worsening the overall trapping efficiency. Conversely, lower flow rates (around Re 0.01) led to longer seeding time, thus enhancing the possibility for cells to aggregate within the perfused suspension and consequently either to be trapped in clusters or to block channels. Noteworthy, at the selected flow rate $(0.2 \mu \mathrm{l} / \mathrm{min})$, the occurrence of a chamber entrapping multiple cells was significantly lower than the percentage of single cell filled chambers. Such multiple cell trapping phenomena were also mainly related to cells aggregation before entering the chamber, rather than to the sequential entrapment of multiple cells.

Cells trapped within the device and cultured up to 3 days, exhibited excellent viability and proliferative activity, proving the proposed approach compatible for isolating and culturing adherent single cells within compartmentalized chambers. Moreover, the chambers configuration exhibited enough space for further cell proliferation, which is a fundamental requirement for further transfection experiments. To the best of our knowledge, the preliminary results showed in this study also demonstrated for the first time the possibility to transfect adherent single cell by means of gene vectors within a microfluidic device.

The potentiality of the presented device to culture individual adherent cells in defined spatial configuration and in a high-throughput fashion further allowed for easily tune the chemical environment around them. Indeed, throughout the innovative transfection strategies development stage, the possibility to deliver controlled combinations of vectors in a spatio-temporal controlled manner is crucial for obtaining fast and reliable results. For this purpose, the integrated serial dilution generator was ad hoc computationally designed for mixing both soluble factors and non-diffusive entities, which essentially are nano-to-micrometric polyplexes and lipoplexes. Numerical modeling showed that the implementation of herringbone structures within the layout was able to increase the mixing efficiency of large particles even at low Reynolds numbers $(\operatorname{Re}=1)$, and therefore such structures were integrated within the final platform. To the best of our knowledge, we here experimentally demonstrated for the first time the generation of linear patterns of non-diffusive particles within a microfluidic device. By injecting viral gene delivery vectors, the establishment of such gradient was quantified through the analysis of the DNA content at each outlet of the six cell culture units. Furthermore, compared to diffusion-based serial dilution generators, the proposed chaotic SDG allowed obtaining reliable linear distribution of polyplexes while maintaining relatively low pressure drops. The integration of this element thus allows extending the potentiality of the device and/or the chaotic SDG module itself for high-throughput screening over a wide range of molecules and particles.

In conclusion, we integrated all the required functionalities for the optimization of non-viral gene transfection protocols within a single microfluidic platform, allowing for testing multiple conditions (e.g. analyzing and comparing the transfection effect of delivering different polyplex doses, over a variety of dynamic conditions, in diverse culture or buffer media, and in different cell types) in an automatic and highthroughput fashion. Preliminary transfection experiments showed the actual possibility to achieve transfection of single cells, thus allowing for directly monitoring the gene transfer efficiency and cytotoxicity by means of an "on-off" investigation at the single cell level. A more exhaustive study is underway in our laboratories to exploit the full potential of this microfluidic device in gene delivery experiments.

\section{Acknowledgements}

This work was partially funded by Fondazione Cariplo, grant no. 2012-0891 and by MIUR - FIRB 2008 Futuro in Ricerca, grant no. RBFR08XH0H. The authors also thank Mara Licini for her invaluable help during the microfluidic platform preliminary validation.

\section{Notes and references}

${ }^{a}$ Department of Electronics, Information and Bioengineering, Politecnico di Milano, Milano, Italy. E-mail: marco.rasponi@polimi.it.

${ }^{b}$ Department of Chemistry, Materials, and Chemical Engineering "G. Natta", Politecnico di Milano, Milano, Italy.

${ }^{c}$ Research Unit Milano Politecnico, National Interuniversity Consortium of Materials Science and Technology - INSTM, Milano, Italy.

${ }^{d}$ Centro Interuniversitario di Ricerca di Biotecnologie Proteiche "The Protein Factory", Politecnico di Milano, CNR-ICRM Milano, and Università degli Studi dell'Insubria, Milano, Italy 
1. D. Pezzoli, R. Chiesa, L. De Nardo and G. Candiani, Journal of applied biomaterials \& functional materials, 2012, 10.

2. S. Mehier-Humbert and R. H. Guy, Advanced Drug Delivery Reviews, 2005, 57, 733-753.

3. V. Vijayanathan, T. Thomas and T. J. Thomas, Biochemistry, 2002, 41, 14085-14094.

4. C. E. Thomas, A. Ehrhardt and M. A. Kay, Nature Reviews Genetics, 2003, 4, 346-358.

5. D. Pezzoli and G. Candiani, Journal of Nanoparticle Research, 2013, 15, 1-27.

6. A. Schmid, H. Kortmann, P. S. Dittrich and L. M. Blank, Current Opinion in Biotechnology, 2010, 21, 12-20.

7. S. Lindström and H. Andersson-Svahn, Biochimica et Biophysica Acta (BBA) - General Subjects, 2011, 1810, 308-316.

8. H. Wang, H.-W. Chen, M. L. Hupert, P.-C. Chen, P. Datta, T. L. Pittman, J. Goettert, M. C. Murphy, D. Williams, F. Barany and S. A. Soper, Angewandte Chemie International Edition, 2012, 51, 4349-4353.

9. S. Hong, Q. Pan and L. P. Lee, Integrative Biology, 2012, 4, 374-380.

10. K. R. Love, S. Bagh, J. Choi and J. C. Love, Trends in Biotechnology, 2013, 31, 280-286.

11. J. El-Ali, P. K. Sorger and K. F. Jensen, Nature, 2006, 442, 403-411.

12. H. Andersson and A. van den Berg, Sensors and Actuators BChemical, 2003, 92, 315-325.

13. A. R. Wheeler, W. R. Throndset, R. J. Whelan, A. M. Leach, R. N. Zare, Y. H. Liao, K. Farrell, I. D. Manger and A. Daridon, Analytical Chemistry, 2003, 75, 3581-3586.

14. C. Hanke, S. Waide, R. Kettler and P. Dittrich, Analytical and Bioanalytical Chemistry, 2012, 402, 2577-2585.

15. A. Lawrenz, F. Nason and J. J. Cooper-White, Biomicrofluidics, 2012, 6.

16. Y. Yamaguchi, T. Arakawa, N. Takeda, Y. Edagawa and S. Shoji, Sensors and Actuators B-Chemical, 2009, 136, 555-561.

17. D. Di Carlo, N. Aghdam and L. P. Lee, Analytical Chemistry, 2006, 78, 4925-4930.

18. S. Kobel, A. Valero, J. Latt, P. Renaud and M. Lutolf, Lab on a Chip, 2010, 10, 857-863.

19. J.-P. Frimat, M. Becker, Y.-Y. Chiang, U. Marggraf, D. Janasek, J. G. Hengstler, J. Franzke and J. West, Lab on a Chip, 2011, 11, 231-237.

20. N. L. Jeon, S. K. W. Dertinger, D. T. Chiu, I. S. Choi, A. D. Stroock and G. M. Whitesides, Langmuir, 2000, 16, 8311-8316.

21. D. Goula, J. S. Remy, P. Erbacher, M. Wasowicz, G. Levi, B. Abdallah and B. A. Demeneix, Gene Therapy, 1998, 5, 712717.

22. M. Ogris, P. Steinlein, M. Kursa, K. Mechtler, R. Kircheis and E. Wagner, Gene Therapy, 1998, 5, 1425-1433.

23. E. V. B. van Gaal, R. van Eijk, R. S. Oosting, R. J. Kok, W. E. Hennink, D. J. A. Crommelin and E. Mastrobattista, Journal of Controlled Release, 2011, 154, 218-232.

24. D. Pezzoli, F. Olimpieri, C. Malloggi, S. Bertini, A. Volonterio and G. Candiani, PLoS ONE, 2012, 7, e34711.

25. A. D. Stroock, S. K. W. Dertinger, A. Ajdari, I. Mezić, H. A. Stone and G. M. Whitesides, Science, 2002, 295, 647-651.

26. Y. N. Xia and G. M. Whitesides, Angew Chem Int Edit, 1998, 37, 551-575.

27. Z. Dai, T. Gjetting, M. A. Mattebjerg, C. Wu and T. L. Andresen, Biomaterials, 2011, 32, 8626-8634.

28. S.-H. Huh, H.-J. Do, H.-Y. Lim, D.-K. Kim, S.-J. Choi, H. Song, N.H. Kim, J.-K. Park, W.-K. Chang, H.-M. Chung and J.-H. Kim, Biologicals, 2007, 35, 165-171.
29. H. Andersson and A. van den Berg, Current Opinion in Biotechnology, 2004, 15, 44-49.

30. X. Mu, W. Zheng, J. Sun, W. Zhang and X. Jiang, Small, 2013, 9, 921.

31. A. K. White, M. VanInsberghe, O. I. Petriv, M. Hamidi, D. Sikorski, M. A. Marra, J. Piret, S. Aparicio and C. L. Hansen, Proceedings of the National Academy of Sciences, 2011, 108, 13999-14004. 